\title{
Corrigendum: Structural basis of synaptic vesicle assembly promoted by $\alpha$-synuclein
}

\author{
Giuliana Fusco, Tillmann Pape, Amberley D. Stephens, Pierre Mahou, Ana Rita Costa, Clemens F. Kaminski, \\ Gabriele S. Kaminski Schierle, Michele Vendruscolo, Gianluigi Veglia, Christopher M. Dobson \\ \& Alfonso De Simone
}

Nature Communications 7:12563 doi: 10.1038/ncomms12563 (2016); Published 19 Sep 2016; Updated 11 May 2017

In the original version of Supplementary Data 1 associated with this Article, the FastPeakFind.m function was incorrectly attributed to the authors in the function's header. This function is Copyright (C) 2012 Adi Natan, Stanford University (natan@stanford.edu). The HTML version of the Article has been updated to include a revised Supplementary Data 1 with the correct attribution.

In the Supplementary Information file, there are errors in the labelling of the $x$ axis in Supplementary Fig. 11. The $x$ axis label should be 'Extrusion Membrane Pore Diameter (nm)' and the labels 'SUV', 'WT' and 'E46K/K80E' should be '50', '70' and '100', respectively. The correct version of this figure appears as Fig. 1 below.

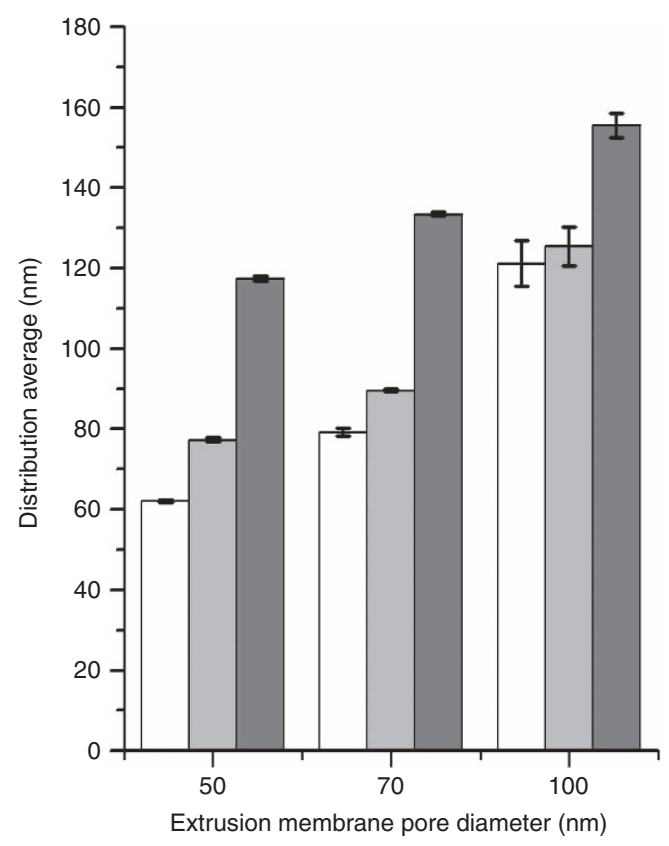

Figure 1 